\title{
IDENTIDAD REGIONAL EN MAGALLANES, SUS EXPRESIONES SIMBÓLICAS Y TERRITORIALES ${ }^{1}$
}

WALTER MOLINA C:

\begin{abstract}
RESUMEN
El presente artículo presenta los resultados más relevantes de un estudio cuantitativo orientado a explorar y describir la identidad regional percibida por los habitantes de la región de Magallanes y Antártica Chilena, a partir del diseño y aplicación de una encuesta de identidad regional aplicada durante el segundo semestre del año 2009. Entre las conclusiones más destacadas del estudio se encuentra la clara conciencia que manifiestan los habitantes de pertenecer a un grupo regional con características propias (86.2\%). Este sentido de pertenencia es alto en todas las provincias de la región, llegando incluso a un 91\% en la provincia de Última Esperanza (Puerto Natales). Por otra parte, entre los elementos simbólicos y territoriales más valorados por los habitantes de la región de Magallanes se pueden mencionar: la valoración positiva de la vida familiar, la seguridad y tranquilidad de la región, la riqueza de su flora y fauna, entre otros aspectos destacados. Lo anterior, presenta relación con lo que desde el punto de vista teórico se sostiene respecto de una identidad regional, es decir, que ésta emerge cuando los actores sociales se sienten profunda y emocionalmente vinculados a un territorio, su historia, tradiciones, paisajes y todas aquellas manifestaciones culturales que dan singularidad a una región o localidad (Amtmann 1997).
\end{abstract}

PALABRAS CLAVE: identidad regional, expresiones simbólicas, territorio, sentido de pertenencia.

\section{REGIONAL IDENTITY IN MAGALLANES, ITS SYMBOLIC AND TERRITORIAL EXPRESSIONS}

\begin{abstract}
This article presents the most notable results from a quantitative study directed to explore and describe the regional identity perceived by the inhabitants of the Region 'Magallanes y Antártica Chilena', from the design and application of a survey of regional identity carried out during the second semester of the year 2009. Among the most outstanding conclusions of the study we find the clear awareness expressed

1 Este artículo forma parte del "Estudio para el Fortalecimiento de la Identidad Regional". Encargado y financiado por el Servicio Gobierno Regional de Magallanes y Antártica Chilena y ejecutado por la Universidad de Magallanes durante el año 2009 (Licitación privada № 1609-11034-B208).

Universidad de Magallanes. Av. Bulnes 01855, Punta Arenas. walter.molina@umag.cl.
\end{abstract}


by people of belonging to a regional group with own characteristics $(86,2 \%)$. This sense of belonging is high in all the provinces of the region, reaching up to 91\% in the province of 'Última Esperanza' (Puerto Natales). On the other hand, some of the territorial and symbolic elements appreciated by the people of the Magallanes region are: the positive appreciation of the family life, safety and tranquility of the region, the riches of its flora and fauna, among others highlighted aspects. This has a relation with what from the theoretical point of view is stated about a regional identity, that is to say, that this emerges when the people feel deep and emotionally linked to a territory, its history, traditions, landscapes and all those cultural expressions which provide singularity to a region or locality (Amtmann, 1997).

KEY WORDS: regional identity, symbolic expressions, territory, sense of belonging.

\section{INTRODUCCIÓN}

La región de Magallanes (territorio americano), ocupa el extremo sudoccidental de Sudamérica y al mismo tiempo confirma la parte más austral del territorio chileno. Comprende la parte meridional de la Patagonia y la sección occidental de la tierra del fuego, y los archipiélagos adyacentes. Se extiende entre los paralelos $48^{\circ} 40^{\prime}$ y $56^{\circ} 30^{\prime}$ de latitud austral y el meridiano $72^{\circ}$ de longitud oeste, abarca una superficie de 132.033,5 kilómetros cuadrados. Además forma parte de esta región el Territorio Antártico Chileno situado a los $90^{\circ}$ y $53^{\circ}$ de longitud oeste. Su población actual es de 152.386 habitantes. Desde un punto de vista geográfico, Martinić (2010) ha destacado la condición de cul de sac continental de la Magallania histórica por ubicarse en el vértice austral del continente americano y reunir allí las aguas de tres océanos, junto a montañas rocosas, glaciares, selvas y llanuras esteparias.

Desde estas singularidades geográficas, la región de Magallanes (y Fuego-Patagonia) emerge a los "ojos" del mundo moderno antes que se conformara Chile como País. Desde este punto de vista, esta región adquiere una importancia estratégica en los momentos en que la cultura de Occidente se esfuerza por ampliar (y descubrir) un vasto territorio sobre el cual se desplegará la primera fase globalizadora del capitalismo moderno.

En este sentido, la historia de la cartografía Magallánica muestra muy claramente cómo a los "ojos de Occidente", de sus navegantes y exploradores, primero aparece la región de Magallanes y la Isla de Tierra del Fuego, antes incluso que el país al que pertenecemos. Este hecho ha sido destacado y puesto en relieve por Martinić (2006) quien, al recibir el premio Bicentenario a las personalidades que han contribuido significativamente al desarrollo de la República de Chile en sus doscientos años de historia, señala lo siguiente:

El verdadero Chile está en Magallanes. No hay porque asustarse. Chile nació en el Sur y fue bautizado en el Norte. En rigor de justicia, así como América debería llamarse Colombia, en la misma forma Chile debería llamarse Magallania; y Magallanes, Chile. Pues la palabra Chile en Aymará significa: donde termina la tierra, y en quechua: donde hace frío. Que todo eso es Magallanes. Magallanes por lo tanto debería ser el verdadero nombre de Chile, y viceversa (Martinić, 2006).

Dado lo anterior, se infiere que los habitantes de Magallanes y Antártica Chilena deben buscar las claves de su desarrollo, actual y futuro, en los diversos y riquísimos aspectos que le dan singularidad a su historia, y a su conformación territorial, única, en el contexto geográfico mundial.

Desde un punto de vista arqueológico, existen variadas evidencias que este vasto territorio fue ocupado tempranamente por grupos de cazadores recolectores terrestres y marítimos desde hace unos 12.000 años antes del presente. A partir de aquella época prehistórica estos grupos humanos iniciaron un largo proceso de adaptación a un territorio marcado por condiciones medioambientales extremadamente adversas para la vida humana. A juicio de Morello y Junge (2010:19) esta impresionante historia de adaptación da cuenta de una templanza humana y evidencia la utilización de estrategias adaptativas que sólo pueden ser comprendidas a partir un conocimiento profundo de las singularidades del territorio y de los rasgos culturales de estos mismos pueblos. De este modo singular, el espacio fue ocupado a lo largo de milenios por una diversidad de pueblos originarios: Kawésqar, Selk`nam, Yaganes, 
Aónikenk, entre otros. Dando lugar a una primera proeza adaptativa en esta austral región del mundo y con ello a una diversidad cultural originaria que data de épocas prehistóricas.

Posteriormente, arriban a la región un importante grupo de inmigrantes provenientes de Chiloé y Europa dando inicio a una segunda proeza adaptativa que logra superar numerosos obstáculos que presenta el entorno venciendo el aislamiento propio de esta zona y mejorando las condiciones de vida. Lo anterior permite establecer asentamientos humanos en Punta Arenas, Puerto Natales, Porvenir y Cabo de Hornos. En este escenario surge un entramado cultural y social rico en matices simbólico-identitarios que dan forma a la sociedad magallánica de mediados del Siglo XIX y cuyos elementos diferenciadores se proyectan hasta la actualidad.

\section{ANTECEDENTES TEÓRICOS}

Los fenómenos de globalización han impactado profundamente la configuración de las sociedades en el Siglo XXI, dando origen a lo que Castells (2003:23) denomina la sociedad red, la cual se caracterizaría por la globalización de las actividades económicas, la organización en redes, la flexibilidad $e$ inestabilidad del trabajo, y la emergencia de una cultura de la imagen y las comunicaciones instantáneas las que ha han dado origen a una virtualidad real construida sobre la base de nuevas formas de trasmisión de mensajes y la conformación de redes de comunicación omnipresentes, interconectadas, diversificadas y diseminadas en los diferentes lugares del planeta. Lo anterior, se manifiesta en la vida social, como una fuerte tensión entre los fenómenos de globalización y los procesos de construcción de identidad social y cultural. Es decir, por una parte, estos fenómenos tienden a estandarizar las conductas de los actores sociales; homogenizando sus producciones culturales, y por otra parte, los actores sociales a través de procesos de construcción de identidad, despliegan y enfatizan las singularidades de sus vidas, sus trayectorias sociales y llaman la atención sobre las particularidades de sus entornos locales.

Desde esta perspectiva, resulta relevante considerar el concepto de identidad social como un aspecto central en el análisis y comprensión de la tensión latente entre procesos de homogenización y diversificación de las identidades en las comunidades humanas. Sin embargo, y dado que desde un punto de vista teórico, la noción de identidad constituye una categoría de análisis compleja y susceptible de múltiples aproximaciones conceptuales, consideramos necesario delimitar su alcance a partir de la revisión de sus principales propiedades constituyentes y sus dinámicas sociales concretas en la vida social contemporánea.

En una primera aproximación, podemos señalar que en la tradición filosófica aristotélica la identidad es concebida como uno de los principios constitutivos del ser y por extensión, como un principio del pensamiento lógico matemático. Al respecto, Larraín (2001) sostiene que el principio ontológico de identidad o de "no contradicción" afirma que todo ser es idéntico a sí mismo y, por lo tanto, una cosa no puede ser y no ser al mismo tiempo. Este enfoque conceptual representaría, según el autor, una concepción esencialista y auto-referencial, no aplicable al estudio de las identidades sociales. Al respecto señala: una mesa es idéntica consigo misma del mismo modo que un ser humano es idéntico consigo mismo aunque la mesa no sea consciente de ello y el ser humano pueda serlo (Larraín: 2001:21). En consecuencia, por largo tiempo la reflexión filosófica redujo el fenómeno de la identidad a un sentimiento de mismidad individual/personal

Considerando las restricciones que presentaría la perspectiva filosófica en el acceso al tema de la identidad; las filosofías modernas comienzan a destacar la reflexividad como elemento crucial en la construcción de la identidad humana (social) y lo hacen tematizando sobre el papel de la autoconciencia y auto-reconocimiento en la configuración de la identidad social. Como reacción a ello, para el pensamiento social contemporáneo resulta necesario definir las identidades como conjuntos de rasgos o cualidades móviles con las cuales las personas o grupos se sienten vinculados. Asimismo, al interior de las ciencias sociales y humanas contemporáneas, comienza a observarse un giro axial en la reflexión sobre los procesos de construcción de identidad, poniendo la noción de pertenencia (o no pertenencia) a una determinada categoría social como centro del nuevo análisis de las identidades. Esta pertenencia a una categoría o subconjunto humano puede ser exteriormente definida o construida por consenso social en el marco de una dialéctica entre similitud y diferencia; es decir, considerando tanto el sujeto 
de la construcción como los otros o la alteridad que la enmarca (Mach, 1993; Salazar, 1998).

En un análisis más empírico, Salazar \& Salazar (1998) sostienen que en los estudios sobre identidad nacional (o regional) realizados recientemente en América Latina, se pueden identificar tres enfoques relevantes sobre la noción de identidad social: a) la identidad concebida como característica objetiva identificable externamente b) la identidad como imagen subjetivas o auto-estereotipo y c) la identidad como identificación intersubjetiva con una categoría (grupal) nacional. Esta última se establecería a través de un complejo proceso de interacción social con determinados elementos que forman parte de la nación. Aún cuando podemos observar diferencias entre los diversos enfoques, no son necesariamente excluyentes entre sí, sino más bien proporcionan elementos analíticos que permiten aproximarnos a las diversas dimensiones de un fenómeno tan complejo como lo es el estudio de las identidades sociales y regionales en el mundo contemporáneo.

\section{IDENTIDAD SOCIAL E IDENTIDAD \\ REGIONAL: UN PROCESO DE CONSTRUCCIÓN SOCIAL}

En el marco del presente estudio y teniendo como referencia algunas de las anteriores conceptualizaciones sobre identidad, es posible abordar la identidad social/cultural considerando el proceso de construcción simbólica que se expresa en las identidades colectivas; lo que da cuenta de una dialéctica irreducible entre sujeto y sociedad. En esta dinámica social se verifica una clara articulación entre los elementos biográficos de los sujetos, las trayectorias sociales de los mismos y los procesos históricos que los condicionan. Por ello, el estudio de las identidades nos remite a comunidades humanas concretas, situadas en un tiempo histórico determinado y localizadas en espacios geográficos concretos. De este modo, resulta relevante, en la demarcación conceptual de la noción de identidad destacar el hecho de que se trata de un proceso de construcción social que va más allá del propio individuo, pero que tiene un vínculo de implicación mutuo con él y los modos colectivos de reaccionar frente al entorno que lo rodea. Desde esta perspectiva, la identidad es un proceso de construcción históricosocial; pues desde esa historicidad los sujetos dan forma a sus aspiraciones, sueños y desafíos que son proyectados en formas de identidades, hacia las nuevas generaciones que a su vez dan continuidad al proceso de construcción histórica de dichas identidades.

En este contexto, en todo proceso de construcción de identidades sociales podemos distinguir tres momentos claramente articulados: Diferenciación, auto-identificación y reconocimiento. Para Therborn (1998) la diferenciación se refiere al proceso de toma de conciencia de la separación del otro o al distanciamiento crítico entre un yo o nosotros (potencial) y el entorno que lo enmarca y condiciona, configurándose la experiencia de la alteridad del otro que a su vez se relaciona con el descubrimiento y construcción del sí mismo. El segundo momento se relacionaría con el proceso de auto-identificación con algo o con alguien, fenómeno descrito por Merton (1966) a partir de cuatro tipos de auto-referencia: origen (de dónde viene la persona: un territorio, un linaje, etc.), residencia (dónde está la persona actualmente) competencia (qué es lo que hace) y valores (en qué cree). Evidentemente, estos cuatro mecanismos de auto- referencia no agotan las posibles fuentes de identidad, pero se constituyen en importantes referentes simbólicos o de producción de sentido para los individuos y grupos que a lo largo de complejos procesos históricos construyen sus identidades culturales.

Finalmente, el tercer momento correspondería al reconocimiento por parte de los otros, instancia determinante para que una identidad funcione y circule socialmente como tal, pues, al no ser reconocida socialmente, y ser perseguida o minusvalorada, se convertiría en un estigma, es decir, en una identidad deteriorada (Goffman, 1998). Para el autor, los movimientos de "autonomía nacional", las corrientes regionalistas o los movimientos indigenistas, constituirían ejemplos de luchas que buscan el reconocimiento sociopolítico de las identidades.

Por otra parte y complementando estas aproximaciones conceptuales, Castells (2003) aborda la construcción social de la identidad desde el entorno sociocultural, el cual marcado por relaciones de poder, daría origen a tres variantes de la construcción de la identidad social: Identidad legitimadora, identidad de resistencia e identidad proyecto. La primera estaría determinada por las instituciones dominantes en la sociedad para extender y racionalizar su 
dominación frente a los actores sociales; la segunda, por aquellos actores sociales que se encuentran en posiciones/condiciones devaluadas o estigmatizadas socialmente, quienes se resisten basándose en principios diferentes $\mathrm{u}$ opuestos a los que imperan en las instituciones de la sociedad. Finalmente, la identidad proyecto, surgiría cuando los actores sociales, basándose en los materiales culturales de los que disponen, construyen una nueva identidad que redefine su posición en la sociedad buscando la transformación de la estructura social en su conjunto.

En consecuencia, en el marco de los actuales procesos de globalización que caracterizan a la sociedad red, en la construcción de las identidades los actores sociales utilizan información tanto de la historia, como de la geografía, de las instituciones como de la memoria colectiva, reordenando en su sentido o re-significando los proyectos culturales vigentes en una determinada estructura social (Castells, 2003)

La identidad regional compartiría con la identidad social y cultural el dinamismo y trabajo constructivo que emerge en la vida social cuando los actores sociales se sienten profunda y emocionalmente vinculados a un territorio, historia, tradiciones, paisajes y expresiones culturales que dan singularidad a una región o localidad (Amtmann, 1997). Por consiguiente, la identidad regional implicaría un compromiso vital con el pasado, presente y futuro de los procesos económico-sociales y culturales que se desarrollan en un espacio territorial delimitado. Potencialmente, esta vitalidad puede convertirse en una fuerza social capaz de impulsar un proyecto de desarrollo compartido por los actores sociales de una determinada región.

La noción de identidad regional se ha ido configurando teniendo como referencia primaria las teorías de la identidad social (Tajfel, 1984, Tajfel y Turner, 1989; Zuñiga y Asún, 2003). Para Tajfel (1984) la identidad social correspondería a una parte del auto-concepto de un individuo que implica el conocimiento de su pertenencia a un grupo social, y que conlleva un significado valorativo y emocional asociado a esta pertenencia. Es decir, la identidad social se estructuraría a partir de la integración de tres dimensiones: cognitiva, evaluativa y emocional involucradas en dicha pertenencia. Basándose en este autor, Zuñiga y Asún (2004) reconceptualizan el término definiendo la identidad regional como una parte del auto concepto de un individuo que surge del conocimiento de su pertenencia a un grupo regional. Específicamente, entendiéndolo como un grupo humano que habita un determinado territorio, el cual tiene una referencia histórica en común y una continuidad sociocultural que la enmarca. Por lo anterior, se concluye que una identidad regional presenta una triple base de sustentación: ecológica, histórica y sociocultural.

Para este estudio resulta relevante destacar aquellos planteamientos que reafirman el valor del proceso de construcción social que implica la noción de identidad, por cuanto los sujetos no sólo reconocen o se identifican con un conjunto de atributos culturales singulares propios de la región; sino que este reconocimiento se sitúan claramente en un contexto histórico, social, cultural y económico más amplio, que es actualizado en cada una de sus expresiones identitarias. Al mismo tiempo, desde esta perspectiva es posible abordar la noción de identidad regional, la cual se construye a partir de la interacción dinámica de los múltiples factores anteriormente señalados y que se interrelacionan en un espacio territorial delimitado, como es la región de Magallanes y Antártica Chilena.

\section{METODOLOGÍA}

Los procedimientos metodológicos utilizados en el estudio corresponden a un enfoque cuantitativo por cuanto se basó principalmente en el diseño y aplicación de una encuesta de identidad, dicha encuesta tiene representatividad regional y provincial y fue aplicada por un equipo de encuestadores que se desplazó a cada una de las secciones de muestreo definidas para la región de Magallanes y Antártica Chilena. Por tanto, este estudio se define como de tipo exploratorio-descriptivo y se orientó a explorar y describir la identidad regional percibida por los habitantes mayores de 18 años que tuvieran una residencia superior a un año en la región de Magallanes y Antártica Chilena.

\section{OBJETIVOS DEL ESTUDIO}

1. Identificar y caracterizar, a través de una encuesta con representatividad regional, los rasgos socioculturales más relevantes de la identidad regional en Magallanes y Antártica Chilena. 
2. Identificar y describir los núcleos simbólicos y expresiones territoriales de identidad en las diferentes provincias y localidades de la región de Magallanes

\section{DISEÑO MUESTRAL}

El diseño muestral del presente estudio es de tipo probabilístico, para ello se tomó como referencia un marco muestral, utilizado previamente para el Programa Integrado de Encuestas de Hogares (PIDEH), el cual fue concebido como un marco de referencia para estudios de propósitos múltiples, donde la conformación de las áreas geográficas del país se presenta dividida en estratos y a su vez éstos, en secciones o conglomerados geográficos (provincias) con información sobre viviendas y población obtenida del Censo de Población y Viviendas 2002. Desde el punto de vista del diseño, la muestra del estudio se definió como probabilística, trietápica y estratificada, con probabilidad de selección proporcional al tamaño de la sección en la primera etapa; una selección sistemática de viviendas en la segunda etapa y finalmente, con selección probabilística de las personas encuestadas.

\section{- Caracterización de la muestra}

La composición final de la muestra lograda en el marco de este estudio fue de 1.488 personas mayores de 18 años. La muestra está compuesta por un $26,5 \%$ de encuestados que residen en zonas rurales y un $73,5 \%$ de residentes en zonas urbanas. En relación a la composición por género, un 39,5\% de los encuestados fueron hombres, y 60,5\% mujeres.

\section{- Composición de la muestra por edades}

Las personas de 40 y más años representan un $57,7 \%$ de la muestra. Por su parte, los sujetos de 21 a 39 años, caracterizados como población adulto joven representan un 26,3\%. Esta distribución por edades también es consistente con la necesidad del estudio de abordar aquella población que potencialmente presentara una mayor permanencia en la región, ya sea porque es "oriundo" o por tener más edad o más años de residencia. El promedio de edad de los sujetos de la muestra fue de 44,2 años de edad.

\section{- Descripción del instrumento}

Para explorar y describir la identidad regional percibida por los habitantes de la región, se diseñó un cuestionario, compuesto de 62 preguntas distribuidas en los siguientes módulos temáticos o ítems: i) información sociodemográfica del entrevistado ii) caracterización del hogar iii) preguntas de opinión e identificación sobre identidad nacional y regional iv) atributos culturales y socio- lingüísticos relevantes en la región v) características de la vivienda y espacios públicos valorados por los habitantes. Cada uno de los ítems estuvo compuesto por preguntas cerradas y abiertas.

\section{PRESENTACIÓN Y DISCUSIÓN DE RESULTADOS}

Los resultados de la encuesta se presentan en correspondencia con aquellos tópicos que desde el punto de vista teórico han sido considerados como los más relevantes al momento de definir una identidad social o regional. En este sentido el primer grupo de datos dicen relación con aquellos elementos que permiten verificar si entre los habitantes de la Región de Magallanes y Antártica Chilena, existe un clara conciencia de pertenencia a un grupo social regional con ciertas características que los diferencia de los habitantes de otras regiones del país. Posteriormente, se presentan los datos que dan cuenta de determinados elementos simbólicos y expresiones territoriales que desde el punto de vista de los encuestados dan singularidad a la región, a sus habitantes y sus diversos territorios o provincias que la componen.

\section{IDENTIDAD Y SENTIDO DE PERTENENCIA REGIONAL}

La primera pregunta general del estudio fue la siguiente: ¿Se considera usted Magallánico? Un $87,4 \%(\mathrm{~N}=1488)$ de los encuestados se considera Magallánico. Un 11\% de ellos, no se considera Magallánico. Y sólo un 1,8\% de los encuestados no responde a esta pregunta inicial.

Considerando que uno de los objetivos de la presente investigación se relaciona con la necesidad de describir las diversas expresiones territoriales de la identidad regional, es relevante analizar las diferencias 
que se presentan en cada una de las provincias. En Puerto Natales el $91 \%$ de los encuestados se considera magallánico. En Punta Arenas, un 86,2\% responde positivamente a esta pregunta, lo mismo ocurre en Porvenir, con $86 \%$, con una leve diferencia, pero en menor medida en Puerto Williams se consideran magallánicos el $76,7 \%$ de la población. Se concluye que en forma homogénea en la población regional existe un alto nivel de conciencia de pertenencia a un grupo regional con identidad propia. Siendo la capital de la Provincia de Última Esperanza (Puerto Natales) quienes más fuertemente se sienten vinculados a la región. Por otra parte, en la provincia Antártica Chilena (Puerto Williams) es donde se verifica la menor proporción de encuestados que se sienten magallánicos y consistentemente con ello, un $23,3 \%$ no se considera parte de esta región. Ello coincide con el alto número de población que reside sólo temporalmente en dicha comuna. Reafirmando este sentido de pertenencia, hemos considerado necesario destacar la percepción de los habitantes respecto del reconocimiento de la identidad regional. Un resultado sobresaliente en los datos de la encuesta es la percepción de los encuestados en torno a la identidad de la región, particularmente frente a la pregunta: ¿La región tiene una identidad propia?. Del total de los encuestados, un alto porcentaje (81\%) responde afirmativamente, mientras que sólo el 10,4\% de ellos no comparte esta afirmación. En este sentido, existe clara conciencia de pertenecer a un grupo regional con características propias y bien definidas y que, además, es un elemento que distingue de otras regiones del país. Respecto de esta pregunta, los resultados por provincia son los siguientes. Ver Fig. 1.

Respecto de esta pregunta, los encuestados residentes en Punta Arenas responden afirmativamente en un porcentaje mayor (85,5\%); por su parte, en Puerto Williams el 79,5\% de los encuestados comparte esta afirmación.

\section{CARACTERÍSTICAS DIFERENCIADORAS DE LA IDENTIDAD REGIONAL EN MAGALLANES}

Como una forma de caracterizar directamente cuales son los rasgos propios de la identidad regional, se preguntó: ¿Cuáles son las características propias de los habitantes de la región de Magallanes? Al respecto, los encuestados expresan sus respuestas del siguiente modo. Ver Fig. 2.

Los resultados obtenidos indican que el rasgo más valorado corresponde a la vida en familia (74,6\%), en segundo lugar el 53\% de los encuestados señala que las características que prevalecen son el ser valientes y sufridos. Por su parte, al abordar los defectos más notorios de los Magallánicos, los encuestados señalan el ser bebedores (25,8\%); en segundo lugar, $y$ vinculado con lo anterior se encuentra el defecto relacionado con el hecho de ser derrochadores y fiesteros (17,4\%). Desde otra perspectiva de análisis, cuando se les pregunta a los encuestados: ¿Cuál es el nivel de valoración que le da a los diferentes aspectos regionales?. Se invita a responder en una escala de 1 (menos valorado)

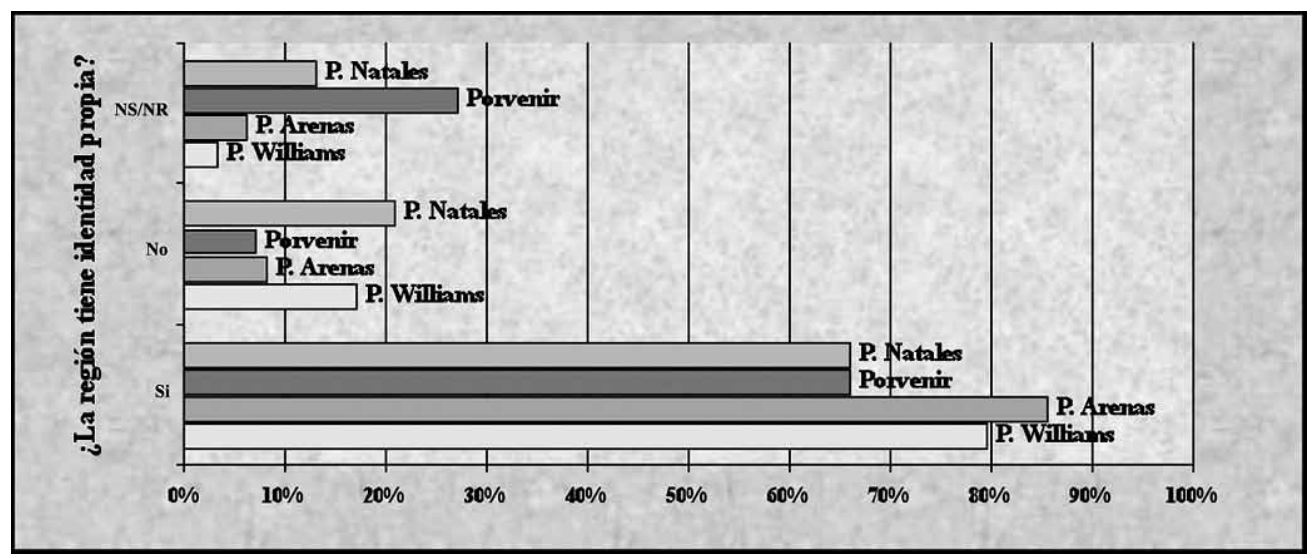

Fig. 1. 


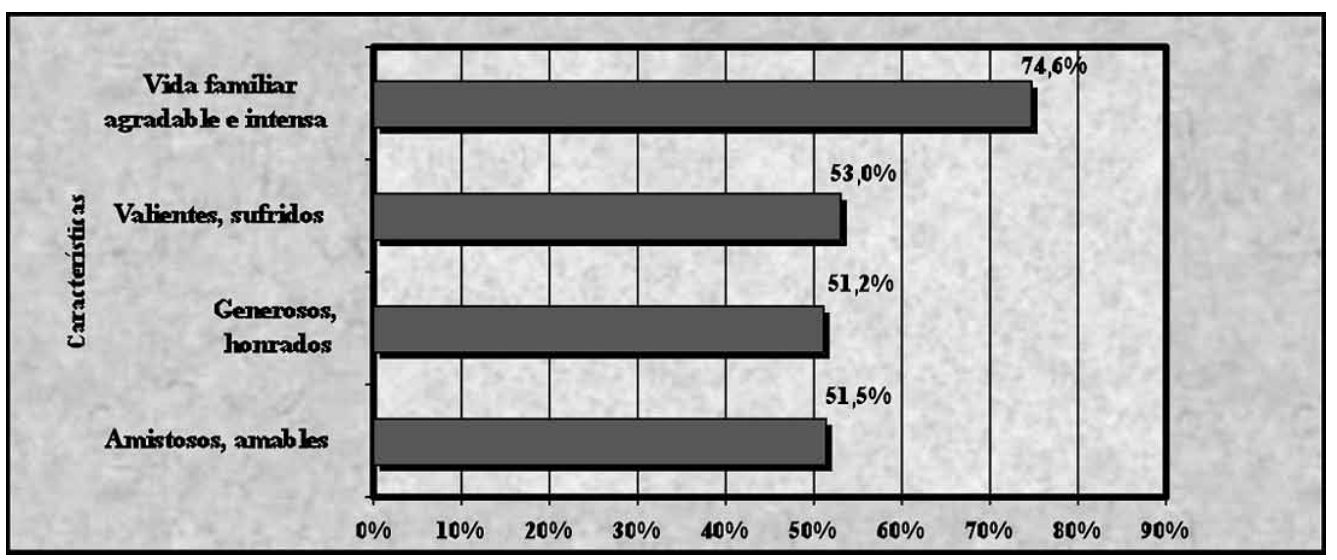

Fig. 2.

a 7 (más valorado) los 1488 encuestados expresan sus respuestas de la siguiente manera. Ver Fig. 3.

Los resultados obtenidos permiten elaborar un ranking con los elementos más valorados por los encuestados y que a su vez se transforman en aspectos marcadores de la identidad regional. En primer lugar, se encuentra la valoración de la riqueza de su flora y fauna $(6,1)$; el segundo lugar, se destaca la forma de ser del magallánico $(6,0)$; el tercer lugar $(5,9)$ es compartido por tres rasgos: temple de los habitantes, sentimiento de pertenencia e historia y cultura de la región; en cuarto lugar, surge la riqueza ganadera $(5,8)$; en quinto lugar, la seguridad y tranquilidad de la vida en la región $(5,6)$; en sexto lugar se encuentran la valoración del proceso de colonización $(5,4)$; en séptimo lugar, se ubican las condiciones laborales y económicas $(5,3)$; y, por último, el aislamiento de la región se considera la característica menos valorada por los encuestados $(4,0)$.

Finalmente, frente a la pregunta: ¿Cuales son las características que más diferencian a la región?. Al respecto, los encuestados expresan sus respuestas en la Fig. 4.

Respecto de las características diferenciadoras, los resultados evidencian que para un importante porcentaje de encuestados (91,6\%) el clima es la característica que más diferencia a la región de otros lugares del país. En segundo lugar, (60,4\%) emergen las singularidades geográficas; en tanto, el 55,5\% de los encuestados señala la extensión o vastedad del territorio como el principal elemento diferenciador. Finalmente, el 47,3\% de los encuestados señala a la historia regional dentro de las principales diferencias con otras regiones del país. La valoración de procesos históricos singulares de

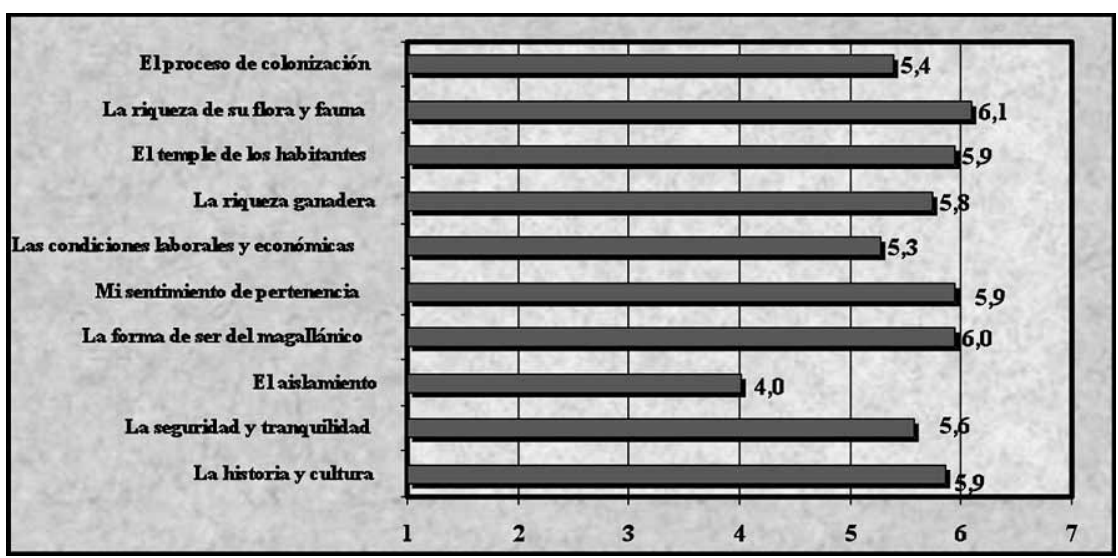

Fig. 3. 


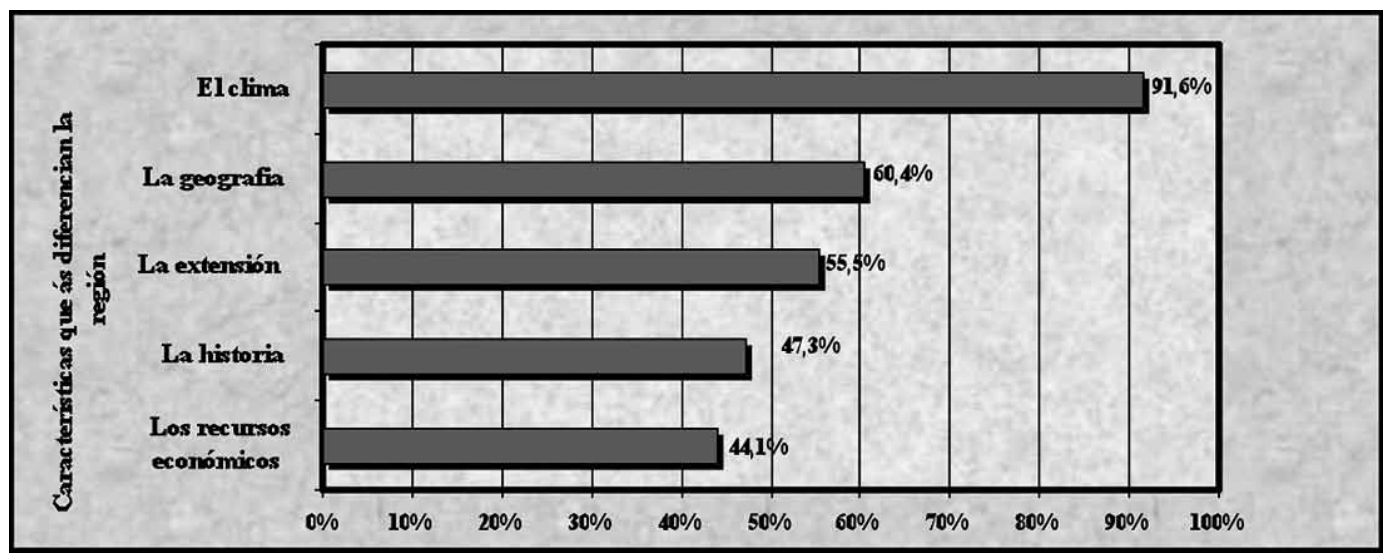

Fig. 4.

la historia regional; tales como, la presencia de pueblos originarios de la región, las dinámicas del asentamiento humano, la singularidad del flujo de población inmigrante, ilustra y realza el sentido de pertenencia regional que tienen los habitantes de la región de Magallanes.

\section{CONCLUSIONES}

Uno de las conclusiones más relevantes del estudio, dice relación con la identificación de los elementos del entorno geográfico y territorial característicos de la región de Magallanes, los que han sido simbolizados de un modo particular por sus propios habitantes. Es decir, aspectos como el clima, los accidentes geográficos o extensión y vastedad del territorio han sido reelaborados simbólicamente por los actores sociales dando origen a una identidad regional claramente definida. En este contexto, cabe destacar lo señalado por Larraín (2001) que así como el ser humano es un ser histórico, por cuanto vive y se desarrolla en una dimensión temporal, de igual forma, es un ser territorial; por cuanto organiza su vida (social) en espacios delimitados dotándolos de significado. De este modo, los sujetos del estudio se proyectan simbólicamente en su territorio de residencia, es decir, territorializan un espacio geográfico; dándole sentidos y significados que finalmente contribuyen de modo importante a la configuración de la identidad regional.

Por otra parte, se concluye que entre los habitantes de la región existe una clara conciencia de formar parte de un grupo social diferenciado en el contexto de la población chilena. Al respecto, es relevante destacar que un alto porcentaje (90\%) de sus habitantes se autodefine como "magallánico". Este sentido de pertenencia regional se expresa en la identificación y alta valoración de elementos singulares del territorio, de la historia regional y rasgos socioculturales específicos. En esta perspectiva de análisis, los encuestados valoran positivamente esta pertenencia, de este modo, sus habitantes se identifican con una serie de atributos personales, tales como: valientes, sufridos, honrados, amistosos, generosos y que caracterizan aspecto muy importante de la identidad regional. Igualmente, en forma mayoritaria se destaca la riqueza de la vida familiar como un aspecto positivo (77\%) de la región.

En tercer lugar, podemos señalar que la identidad regional en Magallanes, específicamente el sentido de pertenencia, es una característica homogéneamente distribuida entre los habitantes de las cuatro provincias que componen el territorio regional, pues manifiestan un fuerte sentimiento de pertenencia a su tierra (región costumbres y tradiciones). Asimismo, se distinguen del resto de los habitantes del país en cuanto a sus características, tradiciones y otros marcadores que dan cuenta de una fuerte identidad regional. Todos los elementos anteriormente mencionados, cristalizan en una autoimagen positiva que valora los elementos propios de la región.

A partir de los datos presentados en este estudio podemos observar que en el caso de la identidad regional en Magallanes, se evidencia una clara articulación de los tres momentos que la literatura especializada distingue en la conformación de las identidades sociales: diferenciación, auto-identificación 
y reconocimiento. Es decir, los datos indican que una parte significativa de los encuestados se reconoce como parte de un grupo social regional que se diferencia claramente de otros conglomerados regionales que conforman el país. Por otra parte, existen elementos específicos de identificación con la historia regional, su paisaje y especialmente con prácticas culturales específicas; los cuales permiten que esta identidad regional sea reconocida como tal y circule socialmente de modo positivo.

Desde otro ángulo de análisis y conclusiones; efectivamente la identidad regional es la parte del auto-concepto de los habitantes de una región que deriva del re-conocimiento explícito de una pertenencia a un grupo social específico. Donde el elemento clave de esa toma de conciencia, se expresa junto al significado valorativo y emocional que los actores sociales regionales le dan dicha pertenencia. De este modo, los datos proporcionados por la encuesta aplica coinciden con los planteamientos de diversos autores que han profundizado en el estudio de las identidades regionales en Chile (Amtmann, 1997; Larraín, 2001; Zuñiga, y Asún, 2003, Martinić, 2010) respecto a los aspectos o tópicos a los que apelan los actores sociales en el proceso de construir sus identidades sociales. Entre estos marcadores de identidad regional se encuentra una profunda valoración emocional de un territorio, su historia, las tradiciones, paisajes (viento o la nieve), modos de habitar el espacio y todas aquellas manifestaciones culturales que dan singularidad a una región o localidad.

En síntesis, la "identidad magallánica" emerge en el marco de un complejo proceso de construcción histórico-social; pues desde esa historicidad los sujetos han dado forma a sus aspiraciones, sueños, desafíos que son proyectados colectivamente hacia las nuevas generaciones. Se destaca así, la construcción social de la identidad regional, como un modo de reaccionar organizadamente a un medio ambiente que presenta dificultades específicas a la vida humana. Y como estas dificultades originarias (y presentes), permiten construir una identidad sociocultural que coincide con lo que Castells (2008) denomina identidad proyecto, es decir, aquella que surge cuando los actores sociales, basándose en los materiales históricos y culturales de los que disponen, construyen una nueva identidad que redefine su posición en la sociedad global o dominante en un tiempo y un espacio determinado. De un modo u otro, esta construcción social, que llamamos identidad regional, busca la transformación de la estructura social en su conjunto, es decir, desarrollo.

Finalmente, se sostiene que profundizar en el conocimiento de las identidades regionales puede contribuir a (re)valorizar las identidades sociales y culturales como un poderoso motor del desarrollo y de fortalecimiento de un capital social/ simbólico de un inapreciable valor para poner en marcha cualquier proceso de expansión económica, social y humana en diversos territorios locales de un planeta global.

\section{AGRADECIMIENTOS}

Agradezco la asesoría metodológica y colaboración en el tratamiento de los datos al Dr. Mario Sandoval Manríquez y al sociólogo Lester Reyes, ambos profesores de la Escuela de Sociología de la Universidad Católica Silva Henríquez. Asi mismo, todo el reconocimiento a los aportes realizados por Rainer Canales del Instituto Antártico Chileno al desarrollo de este estudio.

\section{BIBLIOGRAFÍA}

AMTMANN, C. 1997. Identidad regional y articulación de los actores sociales en procesos de desarrollo regional. $R e$ vista Austral de Ciencias Sociales, 1: 5-14. Universidad Austral de Chile, Valdivia.

CASTELLS, M. 2003. La era de la información. Economía, sociedad y cultura. El poder de la identidad. Siglo XXI, Madrid.

GOFFMAN, E. 1998. Estigma. La identidad deteriorada. Editorial Amorrortu, Buenos Aires.

LARRAÍN, J. 2001. La identidad chilena. Lom editores, Santiago.

MACH, Z. 1993. Symbols, conflict and identity. State University of New Cork, Albano, NY.

MARTINIC, M. 2003. Región Magallánica: Una identidad bien definida. En: Revisitando Chile. Identidades, Mitos e Historias. Montesinos, S. (Comp.). Cuadernos Bicentenario. Presidencia de la República, Santiago.

MARTINIC, M. 2010 La identidad magallánica, su origen y desarrollo en la historia. En: Identidad regional y desarrollo para Magallanes: pp. 13-15. Universidad de Magallanes, Punta Arenas.

MORELLO, F. y P. JUNGE. 2010. Potenciales aportes de la arqueología prehistórica e histórica a la identidad regional. En: Identidad regional y desarrollo para Magallanes: 16-21. Universidad de Magallanes, Punta Arenas. 
SALAZAR, J.M. 1998. Estudios recientes sobre identidades nacionales en América Latina. Psicología Política 16: 75-93.

TAJFEL, H. 1984. Grupos Humanos y Categorías Sociales. Herder, Barcelona.
ZUÑIGA, C. y R. ASÚN 2003. Identidad regional en un contexto de cambio. Un estudio en la Araucanía, Chile. Psicología Política, 26: 73-92.

ZUÑIGA, C.y R. ASÚN 2004. Diseño y validación de una escala de identidad regional. Revista de Psicología Social, 19(1): 35-49. Fundación Infancia y Aprendizaje, España. 
